\title{
Estimation of algal colonization growth on mortar surface using a hybridization of machine learning and metaheuristic optimization
}

\author{
THU-HIEN TRAN and NHAT-DUC HOANG* \\ Institute of Research and Development, Faculty of Civil Engineering, Duy Tan University, P809 - K7/25 Quang \\ Trung, Danang 550000, Vietnam \\ e-mail: tranthuhien197@gmail.com; hoangnhatduc@dtu.edu.vn
}

MS received 20 September 2016; revised 1 January 2017; accepted 1 January 2017

\begin{abstract}
Estimation of the algal colonization growth on façade structure can provide useful information for the task of building maintenance. This research proposes a machine learning method based on the least squares support vector regression (LS-SVR) for modelling the growth time of the green alga Klebsormidium flaccidum on mortar surfaces. Furthermore, to identify an appropriate set of the LS-SVR hyper-parameters, the flower pollination algorithm (FPA) is employed as an optimization technique. The characteristics of the mortar samples, including surface roughness, porosity, surface $\mathrm{pH}$, carbonated condition and type of cement, are employed as input factors for the analysing process. This study relies on a dataset that records 539 laboratory experiments to establish a hybrid model of the LS-SVR and the FPA. The cross-validation process reveals that the proposed method can successfully capture the functional relationship between the algal colonization growth and its influencing factors with a satisfactory outcome (the coefficient of determination $R^{2}=0.94$ and the root mean square error RMSE $=4.55$ ). These facts demonstrate that the hybrid model is a promising tool for assisting the decision-making process in building maintenance planning.
\end{abstract}

Keywords. Alga; biodegradation; least squares support vector regression; flower pollination algorithm; machine learning.

\section{Introduction}

Algae can be transported by wind as spores, fungi, pollens or other forms of particles and diffuse on the building façades rendered with mortars $[1,2]$. In many circumstances, the biological growths bring about various harms to the building. An obvious consequence of algal colonization is that it facilitates other microorganisms to inhabit the building façade. Gradually, the biofilms become more diverse and complex microbial biocoenoses, which include cyanobacteria, heterotrophic bacteria and fungi, are established [3].

These biofilms are responsible for the chemical and physical alterations of the building surface and cause various undesirable effects to the building structure [4]. For instances, extrapolymeric substances may solubilize components of the building materials and damage the substrate through repeated wetting and drying cycles [5]; filamentous microorganisms may penetrate the building material and detach its particles [6].

Additionally, these micro-organisms enhance the water retention of the substrate and lead to reduction of the energy efficiency of buildings [7-9]. The moisture ingress

*For correspondence is responsible for both physical and mental health problems for residents [10, 11]. Considering the building aesthetics, algal growths recognizably affect the façade surface by discolouration and the adherence of dirt particles. The colonization of algae often facilitates the presence of complex crusts and patinas that are very difficult to be eliminated [12]. Hence, the occurrence of microbial growths on the building facade can lead to a significant economic loss for the building owner due to the costs of maintenance and repair [13].

In many situations, the development of algal colonization should be properly monitored and seriously controlled to avoid harmful consequences to the service quality of buildings. The intervention of algal colonization on building envelopes may subsume the approaches of cleaning and preventive treatments. Cleaning can be done with pressured water, with chemicals, using abrasive methods or by laser [14]. Since pressured water washing is effective only for removing thin ingrained soiling from material surfaces, chemical and abrasive cleaning methods are usually required to deal with severe biofilms. The preventative treatments usually involve the application of water repellents and biocides to fend off microbial growth. However, these chemicals may be washed out or can be photolytically and microbially degraded [15]. Since different measures 
require different expenses and each one is appropriate for certain development phases of the algal colonization on the building surface, better understanding and forecasting of the algal growth can be very helpful for building maintenance.

As a consequence, various studies [16-22] have investigated the colonization growth of algae on building surfaces. Besides environmental factors, the rate of algal colonization has been shown to be conditional on the bioreceptivity of the material. Bioreceptivity refers to the aptitude of a material to be colonized by living organisms; and it is related to material characteristics that affect the anchorage and growth of organisms [23]. In addition, the material parameters including surface roughness, surface porosity, surface $\mathrm{pH}$, moisture content and structure/texture of the material have been proved to be influencing factors of the algal colonization [21, 22]. Nevertheless, quantitative studies regarding the colonization process of algae, aiming at forecasting the algal growth, have rarely been carried out due to the complex nature of the problem at hand.

Machine learning, which possesses an excellent capability of nonlinear modelling, can provide viable alternatives for quantitative modelling of algal colonization process. This study extends the body of knowledge by introducing a machine learning method for growing of algae on mortar surface. It is noted that the proposed approach is constructed by an integration of the least squares support vector regression (LS-SVR) [24] and the flower pollination algorithm (FPA) [25].

The LS-SVR, which possesses a desirable learning generalization and a fast computation, has been proved to be very capable for function approximation [26-28]. Moreover, since a successful learning of the LS-SVR requires an appropriate setting of its hyper-parameters (i.e., the regularization and the kernel function parameters) [29, 30], this study utilizes the FPA, a newly established metaheuristic, for assisting the LS-SVR learning phase. The rest of the paper is organized as follows. The research method is described in the second section. The proposed framework for optimizing the hyper-parameter selection of the LSSVR is presented in the third section. The following section reports the experimental results. Conclusions of this study are stated in the final section.

\section{Research method}

\subsection{Experimental dataset}

In this study, the dataset, including 539 laboratory experiments on the green alga Klebsormidium flaccidum, has been collected from previous works of Tran [16] and Dalod [17]. The growth of the alga on mortar surface was under investigation. Forty-nine mortar mixtures were made of cement, siliceous sand and eventual calcareous filler. The mortar mixture was cast into $50 \times 50 \times 1\left(\mathrm{~cm}^{3}\right)$ expanded polystyrene moulds and stored at $21 \pm 1^{\circ} \mathrm{C}$ and $95 \pm 5 \%$ of relative humidity for 7 days before being cut into $20 \times 8 \times 1\left(\mathrm{~cm}^{3}\right)$ specimens. All mortar samples are made in compliance with the NF-EN 196-1 standard. Such mortars samples were then tested for their algal bioreceptivity. On each mortar type, three specimens were sampled to enhance the experimental reliability.

During the experimental process, the mortar surface was periodically sprinkled by a green algae suspension. It is noted that throughout the experiments, the parameters including the rate of sprinkling, the light intensity, the temperature, the initial concentration of the algal suspension and the inclination angle of the samples were kept constant [21]. In this research, six influencing factors (the surface roughness, porosity, surface $\mathrm{pH}$, permeability, cement type and colonization phase) are employed to model the biological growth of the green alga $K$. flaccidum on mortar surfaces.

The surface roughness $\left(I F_{1}\right)$, which represents the topographic profile of the mortar surfaces, was created by a profilometer. The surface profile was then analysed to derive the arithmetic mean of the absolute values of height deviations compared to an average line [31]. The porosity $\left(I F_{2}\right)$ is the ratio of open pore volume to the total volume of materials; this parameter governs the water absorption and the water retention of the material and was measured by mercury intrusion porosimetry. The surface $\mathrm{pH}\left(I F_{3}\right)$ is known to be a crucial factor that affects the settlement and colonization of the alga; this parameter indicates the alkalinity level of the mortar surface, which is measured by a surface electrode.

The permeability $\left(I F_{4}\right)$ affects the material's ability to transmit water; this feature is conditional upon the total porosity, tortuosity, connectivity and distribution of the pores. It is noted that two commonly used cements, an ordinary Portland cement (OPC) that is rich in calcium silicate and an aluminate cement (CAC) that is rich in calcium aluminate, were employed to make the mortar samples. The factor of cement type $\left(I F_{5}\right)$ takes into account the effect of these two cements: $I F_{5}=0$ for the case of the $\mathrm{OPC}$ and $I F_{5}=1$ for the case of the CAC.

In addition, to quantify the colonization growth of the algae, their colonization phase $\left(I F_{5}\right)$ was recorded and employed as an influencing factor. The colonization phase was recorded from the latency phase to the final phase of the alga. These two phases correspond to the time periods required by the alga to cover $0.5 \%$ and $100 \%$ of the sample surface, respectively. The former notifies the moment when the fouling began to appear; the latter indicates the moment when the fouling has colonized the whole surface. In addition to these two extremes, nine intermediate periods of time for the alga to cover from $10 \%$ to $90 \%$ of the mortar surfaces with the interval of $10 \%$ are also recorded during the experimental process.

Figure 1 demonstrates three specimens when (a) $0.5 \%$, (b) $50 \%$ and (c) $100 \%$ of the mortar surfaces are colonized by the green alga K. flaccidum. Furthermore, the 

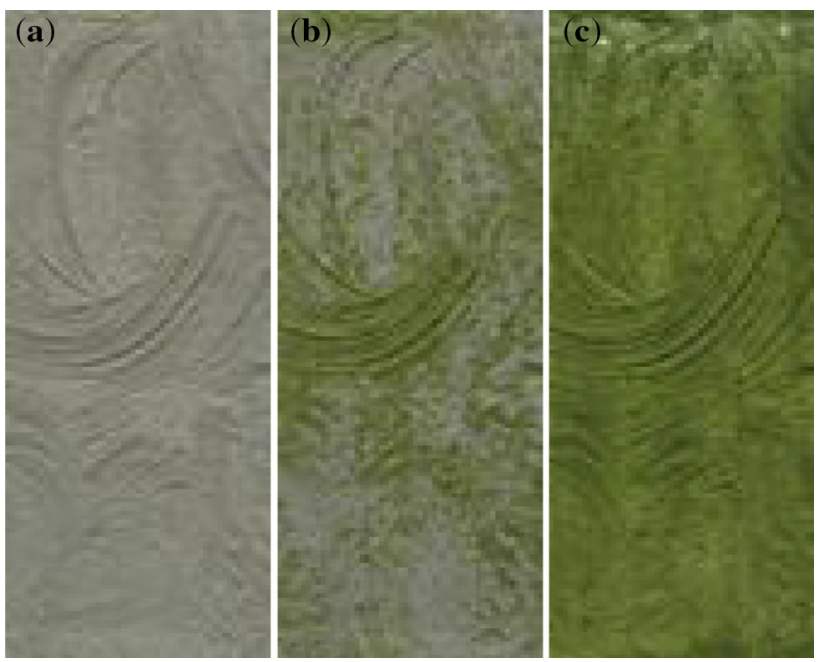

Figure 1. Illustration of mortars colonized by the green alga: (a) $0.5 \%$ of surface coverage; (b) $50 \%$ of surface coverage; (c) $100 \%$ of surface coverage.

colonization time $(Y)$, which is the period of time (measured in day) required for the alga to achieve colonization phases (from $0.5 \%$ to $100 \%$ ), is employed as the modelling output $(Y)$. The statistical descriptions of the colonization time as well as the six aforementioned influencing factors within the dataset are provided in table 1 .

\section{$2.2 L S-S V R$}

The LS-SVR is a machine learning technique, which is established on the principle of structural risk minimization [24, 32]. This learning approach has been successfully utilized to solve various complex problems [29, 33-37]. One notable advantage of the LS-SVR is that the model can be trained very fast since its training process requires solving only a set of linear equations.

The LS-SVR's model establishment boils down to solving the following optimization problem [24, 38]:

$$
\begin{array}{ll}
\operatorname{minimize} & J_{p}(w, e)=\frac{1}{2} w^{T} w+\gamma \frac{1}{2} \sum_{k=1}^{N} e_{k}^{2} \\
\text { subject to } & y_{k}=w^{T} \phi\left(x_{k}\right)+b+e_{k}, \quad k=1, \ldots, N
\end{array}
$$

where $e_{k} \in R$ is an error variable; $\gamma>0$ represents a regularization parameter that quantifies the penalty imposed to data points that deviate from the regression function.

To solve this problem, the Lagrangian is formulated as follows:

$$
L(w, b, e ; \alpha)=J_{p}(w, e)-\sum_{k=1}^{N} \alpha_{k}\left\{w^{T} \phi\left(x_{k}\right)+b+e_{k}-y_{k}\right\}
$$

where $\alpha_{k}$ denotes Lagrange multipliers.

The Karush-Kuhn-Tucker conditions for optimality can be applied by differentiating the Lagrangian function $L(w$, $b, e, \alpha)$ with the variables in the following manner:

$$
\left\{\begin{array}{l}
\frac{\partial L}{\partial w}=0 \rightarrow w=\sum_{k=1}^{N} \alpha_{k} \phi\left(x_{k}\right) \\
\frac{\partial L}{\partial b}=0 \rightarrow \sum_{k=1}^{N} \alpha_{k}=0 \\
\frac{\partial L}{\partial e_{k}}=0 \rightarrow \alpha_{k}=\gamma e_{k}, \quad k=1, \ldots, N \\
\frac{\partial L}{\partial \alpha_{k}}=0 \rightarrow w^{T} \phi\left(x_{k}\right)+b+e_{k}-y_{k}=0, \quad k=1, \ldots, N
\end{array}\right.
$$

After solving the linear system in Eq. (3), the LS-SVR model for function approximation is shown as follows:

$$
y(x)=\sum_{k=1}^{N} \alpha_{k} K\left(x_{k}, x_{l}\right)+b
$$

where $\alpha_{k}$ and $b$ are the solution to the linear system; $k$ and $N$ represent, respectively, the index and the total number of data points in the training set; $x_{k}$ and $x_{l}$ are input patterns in the training and testing set, respectively. $K(\cdot)$ denotes a kernel function; the commonly used Radial Basis Function (RBF) kernel function is shown as follows:

$$
K\left(x_{k}, x_{l}\right)=\exp \left(-\frac{\left\|x_{k}-x_{l}\right\|^{2}}{2 \sigma^{2}}\right)
$$

\begin{tabular}{|c|c|c|c|c|c|c|c|}
\hline Statistics & $\begin{array}{c}I F_{1} \\
\text { Roughness } \\
(\mu \mathrm{m})\end{array}$ & $\begin{array}{c}I F_{2} \\
\text { Porosity } \\
(\%)\end{array}$ & $\begin{array}{c}I F_{3} \\
\mathrm{pH} \\
\text { surface }\end{array}$ & $\begin{array}{c}I F_{4} \\
\text { Permeability }\left(10^{-10}\right. \\
\mathrm{m} / \mathrm{s})\end{array}$ & $\begin{array}{c}I F_{5} \\
\text { Cement } \\
\text { type }\end{array}$ & $\begin{array}{c}I F_{6} \\
\text { Colonization phase } \\
(\%)\end{array}$ & $\begin{array}{c}Y \\
\text { Colonization time } \\
\text { (day) }\end{array}$ \\
\hline Min. & 23.50 & 10.64 & 8.70 & 4.05 & 0.00 & 0.50 & 2 \\
\hline Mean & 104.30 & 25.28 & 10.02 & 19.13 & 0.33 & 50.05 & 27 \\
\hline Median & 61.75 & 31.00 & 9.05 & 17.07 & 0.00 & 50.00 & 20 \\
\hline Std. dev. & 75.70 & 10.17 & 1.24 & 14.46 & 0.47 & 31.55 & 20 \\
\hline Max. & 281.25 & 37.18 & 12.15 & 53.07 & 1.00 & 100 & 96 \\
\hline
\end{tabular}

where $\sigma$ denotes the kernel function parameter, which affects the smoothness of the approximated nonlinear function.

Table 1. Statistical description of the dataset. 


\subsection{FPA}

The FPA [25] is a metaheuristic that has the inspiration from the characteristics of the flower pollination process. In the natural world, pollination is the process by which pollen is transported from the male section (anther) to the female section (stigma) of the plant. Typically, there are two means of pollination: biotic and abiotic [39]. The first means of pollination is often achieved by the assistance of pollinators such as insects, birds and other animals. The second means of pollination does not involve pollinators; instead of that, wind and water flow are employed to deliver pollen.

In addition, pollination can be categorized into two major forms: self-pollination and cross-pollination [40]. In crosspollination, pollen of a flower is transferred to a flower of a different plant. Meanwhile, the second form of pollination is the process of transferring pollen from one flower to another flower of the same plant. It is noted that in biotic pollination and cross-pollination, pollen can be transported to distant areas since pollinators such as bees and birds can travel long distances.

Inspired from such features of the pollination process, the FPA is formulated as an optimization algorithm in which biotic pollination and cross-pollination act as global search; abiotic pollination and self-pollination are utilized as local search (see figure 2) [25]. Compared with other nature-inspired algorithms, the FPA possesses a promising capability of global search [41-44]. In the FPA, a solution $x$ in the search space represents a flower. For the purpose of locating the optimal solution, pollination, in either global or local forms, is considered to be successful if the pollen from the solution $x_{i}$ is transferred to the solution $x_{j}$ with a better fitness. Because the moving patterns of bees and birds can have Levy flight [40,45] behaviour with fly distance steps following a Levy distribution, the global search of the algorithm may benefit from Levy flights. Furthermore, the local and global pollinations can be controlled by a switch probability $p$. Experimentally, Yang [25] recommended that $p$ can be set as 0.8 .

The Levy-flight-based global pollination can be mathematically stated as follows:

$$
x_{i}^{\text {trial }}=x_{i}^{t}+L \times\left(x_{i}^{t}-x_{\text {best }}\right)
$$

where $x_{i}^{t}$ and $x_{i}^{\text {trial }}$ are solution vector $x_{i}$ at iteration $t$ and a trial solution, respectively; $x_{\text {best }}$ denotes the current best solution. The parameter $L$ dictates the length of pollen travelling distance. Herein, the Levy flight is employed to mimic flying patterns of pollinators. $L$ is a random variable drawn from a Levy distribution.

The local pollination as a means of local search can be expressed as follows:

$$
x_{i}^{\text {trial }}=x_{i}^{t}+\varepsilon \cdot\left(x_{i}^{t}-x_{j}^{t}\right)
$$

where $x_{i}^{t}$ and $x_{j}^{t}$ are pollens produced by different flowers of the same plant species; $\varepsilon \in[0,1]$ is a random number generated from a uniform distribution.

\section{Optimizing the LS-SVR with the FPA}

In practical situations, the task of model selection in machine learning requires the determination of a set of hyper-parameters. Generally, the hyper-parameters are selected to minimize a cross-validation-based cost function. This task has been shown to be very tedious; the reason is that the cost functions can be non-smooth and may feature multiple local optimums. A conventional method for model selection is to rely on a grid search over the parameter space. Nevertheless, the grid search approach features two critical difficulties: (i) it may require a huge computational cost due to the combinatorial explosion of grid points and (ii) the discrete grid points cannot exhibit the continuous nature of the hyper-parameter space [46].

As mentioned earlier, the LS-SVR training phase necessitates two hyper-parameters: the regularization parameter $\gamma$, which controls the penalty imposed to data points located far from the regression function, and the kernel function parameter $\sigma$, which adjusts the regression function's smoothness. These two search parameters are in a continuous space and the number of pairs of such parameters is infinite. To tackle this problem, this research employs the FPA as a metaheuristic to conveniently seek for an appropriate set of the LS-SVR hyper-parameters. Since very large value of $\gamma$ and very small value of $\sigma$ can lead to overfitted models, the regularization and kernel constants in this study are allowed to be varied between 0.001 and 1000 .

This section of the article describes the concept of utilizing the FPA for optimizing the learning process of the LS-SVR. It is noted that the LS-SVR is implemented via the LSSVMlab toolbox [32]; the FPA is adopted from the Matlab code provided by Yang [40]. The overall framework that integrates the FPA and the LS-SVR is constructed in Matlab environment by the authors. The overall flowchart for optimizing the LS-SVR with the FPA (see figure 3) is described as follows:

(1) Data separation process: As mentioned earlier, in this study, the dataset consists of 539 laboratory experiments and features six influencing factors (the surface roughness, porosity, surface $\mathrm{pH}$, permeability, cement type and colonization phase). Since the regularization and kernel function parameters significantly affect the learning phase of the LS-SVR, these two parameters should be set properly via a model selection process. To achieve this goal, the whole dataset is randomly divided into 10 mutually exclusive folds. Accordingly, the average model performance obtained from the 10-fold cross-validation is use to quantify the appropriateness of each pair of hyper-parameters. In this way, the bias in model selection can be minimized.

(2) Hyper-parameter initialization: At the first iteration, the two hyper-parameters of the LS-SVR $(\gamma$ and $\sigma)$ are randomly generated as follows:

$$
x=L B+U_{[0,1]}(U B-L B)
$$


Generate an initial population of $\mathrm{N}$ flowers randomly

Evaluate population fitness

Identify the best solution $\boldsymbol{x}_{\text {best }}$ in the current population

Define the switch probability $\boldsymbol{p}$

Define the maximum iteration $\boldsymbol{T}_{\boldsymbol{m a x}}$

For $\boldsymbol{t}=1$ to $\boldsymbol{T}_{\max }$

For $i=1$ to $N$

$\boldsymbol{r} \sim U(0,1) / / \boldsymbol{r}$ is a uniform random number

If $\boldsymbol{r}<\boldsymbol{p}$

Generate $L \sim$ Levy

Perform global pollination: $x_{i}^{\text {trial }}=x_{i}^{t}+L \cdot\left(x_{i}^{t}-x_{\text {best }}\right)$

else

Perform local pollination: $x_{i}^{\text {trial }}=x_{i}^{t}+\varepsilon \cdot\left(x_{i}^{t}-x_{j}^{t}\right)$

\section{End if}

$$
\begin{aligned}
& \text { If } f\left(x_{i}^{\text {trial }}\right)<f\left(x_{i}^{t}\right) \\
& x_{i}^{t}=x_{i}^{\text {trial }} \\
& \text { If } f\left(x_{i}^{t}\right)<f\left(x_{\text {best }}\right) \\
& x_{\text {best }}=x_{i}^{t}
\end{aligned}
$$

\section{End if}

\section{End if}

\section{End For}

\section{End For}

\section{Return $x_{\text {best }}$}

Figure 2. The flower pollination algorithm (FPA).

where $x$ denotes a hyper-parameter; $L B$ and $U B$ are the lower and upper boundaries, respectively; $U_{[0,1]}$ represents a uniform random number within the range of 0 and 1.

(3) Training and prediction phases of the LS-SVR models: At this stage, the data folds taken from the first stage (data separation process) are employed to train 10 different LS-SVR models. Each model in turn uses nine data folds as training data and one data fold as testing data. It is noted that all models are trained with the same set of hyper-parameters, which is provided by the FPA.

(4) Fitness evaluation: The objective function, used in the FPA optimization, is designed to quantitatively measure the prediction accuracy of each LS-SVR model accompanying a set of hyper-parameters. In this study, the following fitness function, which measures the average model performance obtained from the 10 -fold cross-validation process, is employed:

$$
\text { min. } f=\frac{\sum_{i=1}^{10} R M S E_{i}}{10}
$$

where $R M S E_{i}$ denotes the testing error, quantified by the root mean square error (RMSE) of the $i$ th LS-SVR model. The RMSE is computed as follows: 


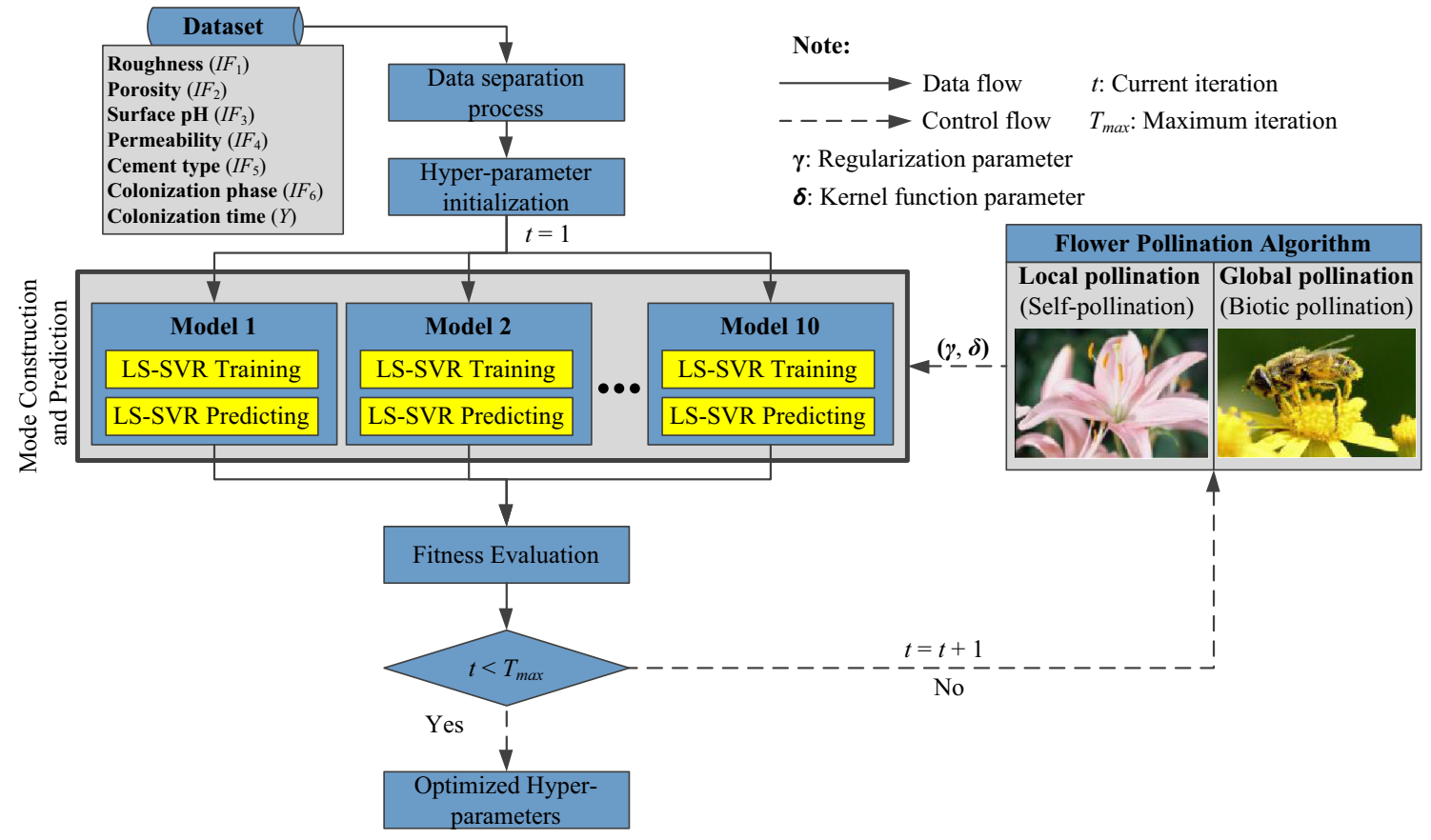

Figure 3. The proposed LS-SVR optimization using the FPA.

$$
R M S E=\sqrt{\sum_{j=1}^{N} \frac{\left(Y_{P}^{j}-Y_{A}^{j}\right)^{2}}{N}}
$$

where $Y_{P}^{j}$ and $Y_{A}^{j}$ represent, respectively, the predicted and actual values of the $j$ th output. $N$ is the number of data samples.

(5) FPA: The FPA is employed to optimize the LSSVR's model selection process. Iteratively, the FPA carries out the global and local pollination phases to guide the population of flowers to better solutions. By evaluating the fitness of each individual, the algorithm discards inferior combinations of $\gamma$ and $\sigma$. Gradually, the FPA allows highquality combinations of these parameters to be passed on the next iterations. The searching process terminates when the current iteration is equal to the maximum iteration number $\left(T_{\max }\right)$. This indicates that the most suitable set of hyper-parameters has been identified by the FPA.

\section{Experimental results}

\subsection{FPA-based optimization result}

In this study, the FPA is employed to autonomously search for a proper set of the LS-SVR's hyper-parameters (the regularization parameter $\gamma$ and the RBF kernel function parameter $\sigma$ ). It is worth noticing that to reduce the bias in assessing the model accuracy, the average RMSE of models (refer to Eq. 9), attained from the cross-validation process, is used to calculate the solutions' healthiness. In this study, considering that the number of searched variables $(\gamma$ and $\sigma$ ) is not large, the number of flowers in the population $(N)$ and the maximum number of iterations $\left(T_{\max }\right)$ are set as follows: $N=10$ and $T_{\max }=300$.

The record of fitness during the FPA optimization progress is illustrated in figure 4. It can be seen that the FPA started from a set of random candidates with relatively inferior fitness and gradually guided the population of flowers towards better solutions; this was reflected by the enhancement of fitness value during the searching process. The algorithm found the best solution $\left(\gamma-=1000, \sigma^{2}=0.58\right)$ with the fitness $f=4.55$ at the 174th iteration; after this moment, no improvement of the fitness has been observed.

Furthermore, figure 5 provides the records of all visited positions of flowers in the search space as well as the positions of flowers at the first and final iterations. At the first iteration, the solutions are randomly scattered over the search space (see figure 5b). Meanwhile, at the final iteration, most of the candidates are clustered around the best found solution. Nevertheless, at this stage, the global pollination operator carries out the explorative search; this is illustrated by a small cluster of solutions in figure $5 \mathrm{c}$.

\subsection{Prediction results and comparison}

To construct and validate the prediction models, the whole dataset, consisting of 539 samples, is randomly divided into two sets: training set $(90 \%)$ and testing set $(10 \%)$. Accordingly, the numbers of samples in these two sets are 485 and 54, respectively. In addition, to confirm the 


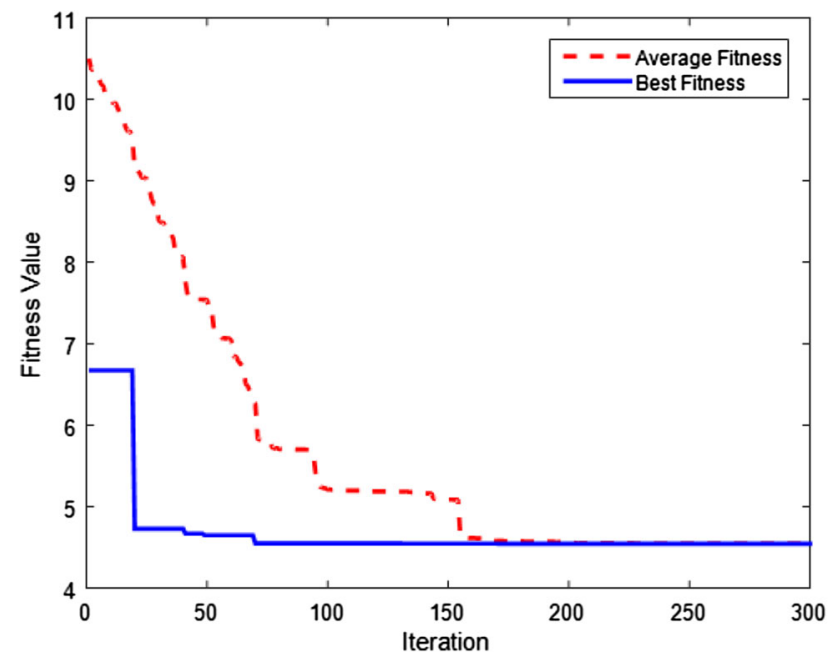

Figure 4. Record of fitness during the FPA optimization.

predictive capability of each model better, a 10-fold crossvalidation process is performed. Accordingly, the whole collected dataset is randomly separated into 10 mutually exclusive folds in which each fold in turn is reserved as testing data and the other nine folds are utilized to train the prediction models. Since all of the data folds are mutually exclusive, the cross-validation process reliably reveals the predictive capability of each modelling method. It is noted that the cross-validation process used in this section is different from the process carried in section 3 of the paper. The former is employed for evaluating model learning result, and the latter has been used for selecting hyperparameters of the LS-SVR.

Moreover, the RMSE and the coefficient of determination $\left(R^{2}\right)$ are employed to express the model's prediction accuracy. The RMSE quantifies the difference between the output values actually observed and the output values obtained from a prediction model after being trained. It is noted that a smaller RMSE indicates a better prediction performance. Besides, the $R^{2}$ demonstrates the proportion of the variability in the output data $(Y)$ explained by the model; and this index indicates how well the constructed model regresses the colonization time of the alga on the influencing factors. $R^{2}=1$ indicates a perfect model. Meanwhile, $R^{2}=0$ implies that there is absolutely no agreement between the model's results and actual outputs.

In this experiment, it is noted that the LS-SVR assisted by the FPA optimization is denoted as LSVR-FPA. Besides the proposed LSVR-FPA, the Levenberg-Marquardt Artificial Neural Network (LM-ANN) [47] and the Multiple Linear Regression (MLR) are employed for benchmarking the prediction results. The MLR is a traditional statistical method applied for exhibiting a linear functional relationship between the predictors and the response variable. Although the MLR cannot render nonlinear mapping, this approach is often employed as a benchmark method for function approximation tasks [48]. Additionally, the LMANN has been successfully applied in modelling complex environmental systems [49-51]. It is noted that the Neural Network Toolbox of Matlab [52] is utilized for implementing LM-ANN models; meanwhile, the MLR model is programmed in Matlab environment by the authors.

Furthermore, to determine the configuration of the LMANN model (the number of neurons, the type of activation functions and the learning rate), a cross-validation process, which is similar to the one employed for identifying the LSSVR's hyper-parameters, has been carried out. The number of neurons in the hidden layer begins with six (which is equal to the number of input variables) and then gradually increases to 30 neurons. Different values of the learning parameter $(0.0001,0.0005,0.001,0.005,0.01,0.05,0.1,0.5$ and 1) of the LM-ANN have also been attempted. Additionally, the experiment has investigated the effect of the (a)

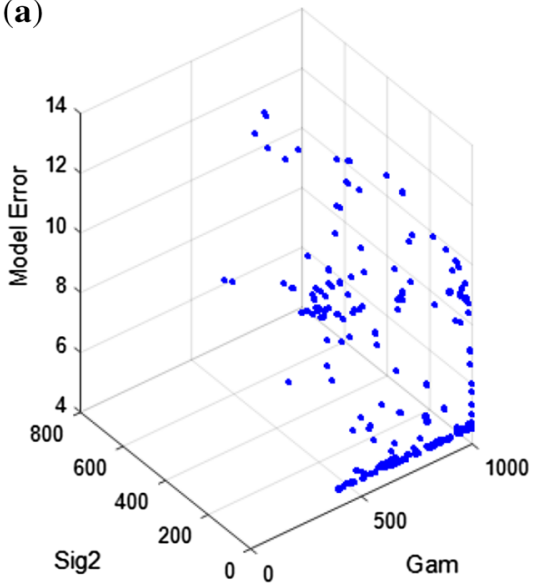

(b)

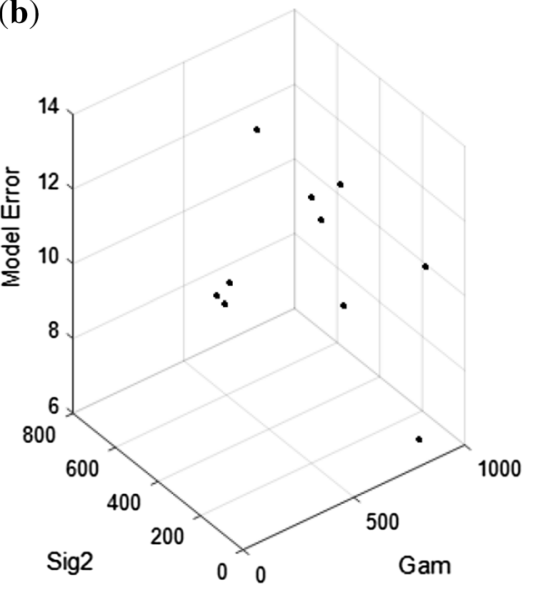

(c)

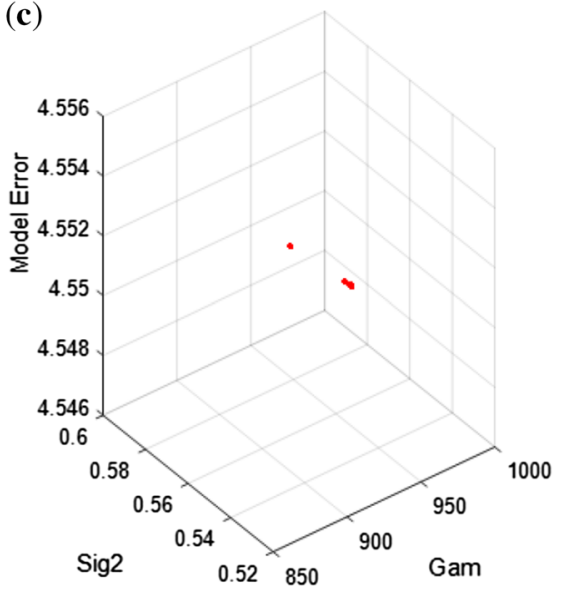

Figure 5. Records of flower positions in the search space: (a) all visited positions; (b) at the first iteration; (c) at the final iteration. 
tan-sigmoid and log-sigmoid activation functions on the performances of an LM-ANN. The number of training epochs is fixed to be 5000. The experimental outcome for the ANN model configuration is that when the number of neurons is 17 , the learning rate $=0.001$ and the log-sigmoid activation function is used, the LM-ANN obtained the best prediction result with the average RMSE $=5.64$.

The experimental outcome of the cross-validation process is reported in table 2. Observably, both machine learning models demonstrate to be very capable tools for fitting the dataset at hand. Moreover, the LSVR-FPA (RMSE $=4.55, R^{2}=0.94$ ) has produced the average predictive performance that is superior to those of the LM-ANN (RMSE $\left.=5.45, R^{2}=0.92\right)$ and the MLR (RMSE $=13.34, R^{2}=0.53$ ). Poor performance obtained from the MLR indicates that the underlying function that governs that mapping between the set of influencing factors (the surface roughness, porosity, surface $\mathrm{pH}$, permeability, cement type and colonization phase) and the colonization time of the alga is possibly complex and highly nonlinear. Such results verify the necessity of applying sophisticated machine learning approaches for approximating the nonlinear function of interest.

Considering the two machine learning models, the LSSVR assisted by the FPA demonstrates better prediction accuracy in both training (RMSE $\left.=2.55, R^{2}=0.98\right)$ and testing processes (RMSE $\left.=4.55, R^{2}=0.94\right)$. The training and testing results of the proposed LSVR-FPA, including all 10 data folds, are shown in figures 6 and 7, respectively. As observed from these two figures, in all data folds of both training and testing phases, the scatterplots of the actual colonization time versus the predicted colonization time demonstrate good fits to the line of best fit. These graphical observations are numerically confirmed by comparatively high values of the $R^{2}$ and low values of the RMSE.

Furthermore, figure 8 provides the scatterplot of residuals versus actual output of colonization time and the distribution of residual summarized from the 10-fold cross- validation process of the LSVR-FPA. It is noted that the residual of a prediction model is defined as the difference between the actual output and the predicted output. Herein, only testing phases of data folds are taken into account. The scatterplot and the residual histogram in figure 8 show that the residual centralizes around zero with a relatively small variance throughout the range of the actual output. In fact, the residual of the LSVR-FPA has a mean of -0.0049 and a standard deviation of 4.6775. In addition, the coefficient of determination $R^{2}$ of the actual colonization time and the residual is 0.0025 , meaning that no pattern can be observed and the variance of the residuals is approximately constant throughout the range of the colonization time. In other words, residuals resulted from the model feature a homogeneous variance. This fact indicates that the LSVR-FPA has generalized the mapping function between the output variable and the influencing variables well with all recorded values of the output; it is evident that no specific range of the colonization time has a different variability from others.

A more detailed analysis of the residuals uncovers that $50.46 \%$ of the residuals are positive and $49.54 \%$ of the residuals are negative. These two values are close to $50 \%$, meaning that the model does not either underestimate or overestimate the algal colonization time. Furthermore, $86.09 \%$ of the residuals are within \pm 5 days of deviation and $96.29 \%$ of the residuals are within \pm 10 days of deviation. Only $3.71 \%$ and $0.74 \%$ of the residuals are beyond \pm 10 and \pm 20 days of deviation, respectively. These facts strongly prove that the proposed LSVR-FPA can deliver accurate and reliable forecasts regarding the algal colonization time.

\section{Conclusion}

This study contributes to the body of knowledge by proposing a machine learning solution for predicting the colonization growth of the green alga $K$. flaccidum on

Table 2. Results of the cross-validation process.

\begin{tabular}{|c|c|c|c|c|c|c|c|c|c|c|c|c|c|}
\hline \multirow[b]{2}{*}{ Model } & \multirow[b]{2}{*}{ Phase } & \multirow[b]{2}{*}{ Indices } & \multicolumn{10}{|c|}{ Fold } & \multirow[b]{2}{*}{ Mean } \\
\hline & & & 1 & 2 & 3 & 4 & 5 & 6 & 7 & 8 & 9 & 10 & \\
\hline \multirow[t]{4}{*}{ LSVR-FPA } & \multirow[t]{2}{*}{ Train } & RMSE & 2.60 & 2.47 & 2.56 & 2.54 & 2.47 & 2.65 & 2.35 & 2.60 & 2.65 & 2.63 & 2.55 \\
\hline & & $R^{2}$ & 0.98 & 0.98 & 0.98 & 0.98 & 0.98 & 0.98 & 0.99 & 0.98 & 0.98 & 0.98 & 0.98 \\
\hline & \multirow[t]{2}{*}{ Test } & RMSE & 3.68 & 6.70 & 4.23 & 4.59 & 5.63 & 3.60 & 5.50 & 4.82 & 3.20 & 3.51 & 4.55 \\
\hline & & $R^{2}$ & 0.96 & 0.83 & 0.95 & 0.92 & 0.94 & 0.97 & 0.94 & 0.95 & 0.97 & 0.97 & 0.94 \\
\hline \multirow[t]{4}{*}{ LM-ANN } & \multirow[t]{2}{*}{ Train } & RMSE & 3.77 & 3.05 & 3.76 & 4.49 & 3.38 & 3.40 & 4.81 & 3.86 & 5.51 & 4.04 & 4.01 \\
\hline & & $R^{2}$ & 0.96 & 0.98 & 0.96 & 0.95 & 0.97 & 0.97 & 0.94 & 0.96 & 0.92 & 0.96 & 0.96 \\
\hline & \multirow[t]{2}{*}{ Test } & RMSE & 4.76 & 6.85 & 5.03 & 5.63 & 5.61 & 5.61 & 8.17 & 4.98 & 3.94 & 3.91 & 5.45 \\
\hline & & $R^{2}$ & 0.94 & 0.82 & 0.93 & 0.88 & 0.94 & 0.92 & 0.86 & 0.94 & 0.95 & 0.96 & 0.92 \\
\hline \multirow[t]{4}{*}{ MLR } & \multirow[t]{2}{*}{ Train } & RMSE & 13.30 & 13.29 & 13.36 & 13.52 & 12.99 & 13.31 & 13.00 & 13.42 & 13.17 & 13.22 & 13.26 \\
\hline & & $R^{2}$ & 0.55 & 0.56 & 0.55 & 0.55 & 0.55 & 0.55 & 0.56 & 0.53 & 0.56 & 0.55 & 0.55 \\
\hline & \multirow[t]{2}{*}{ Test } & RMSE & 13.00 & 13.16 & 12.44 & 10.87 & 15.76 & 12.85 & 15.55 & 11.87 & 14.17 & 13.76 & 13.34 \\
\hline & & $R^{2}$ & 0.55 & 0.35 & 0.57 & 0.57 & 0.53 & 0.56 & 0.50 & 0.68 & 0.40 & 0.56 & 0.53 \\
\hline
\end{tabular}

Bold values indicate important experimental results 

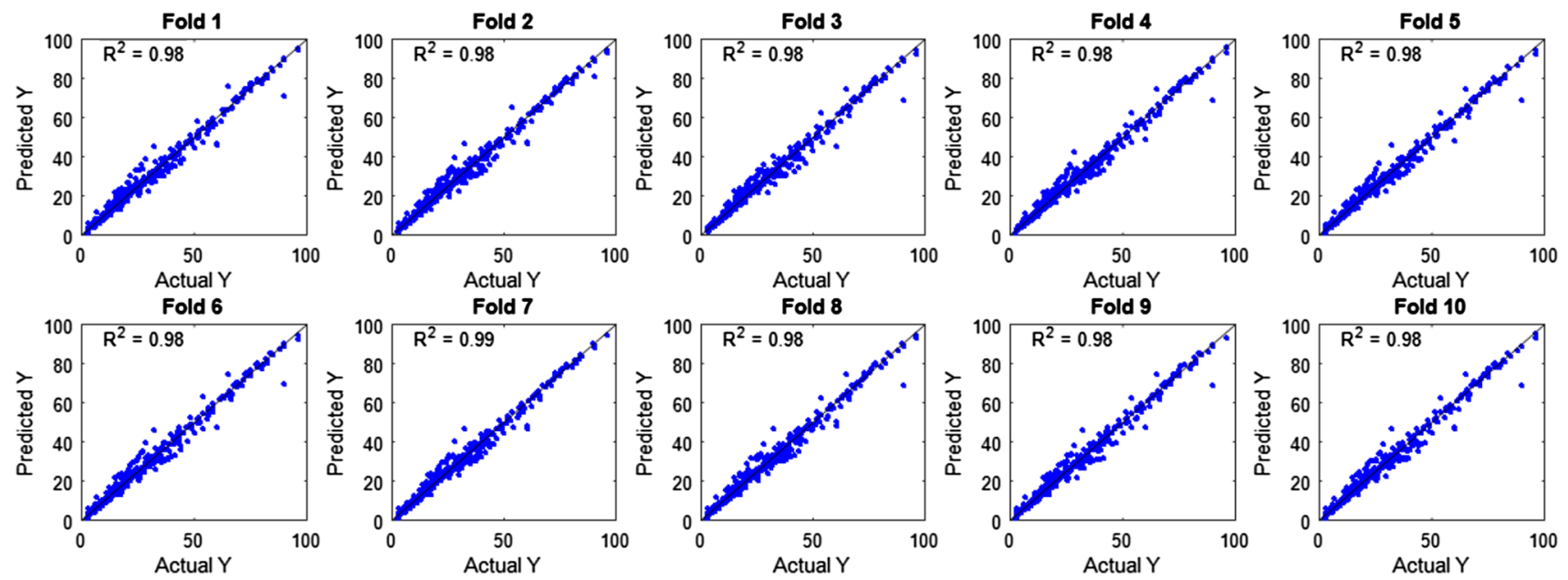

Figure 6. Training results of the LSVR-FPA.
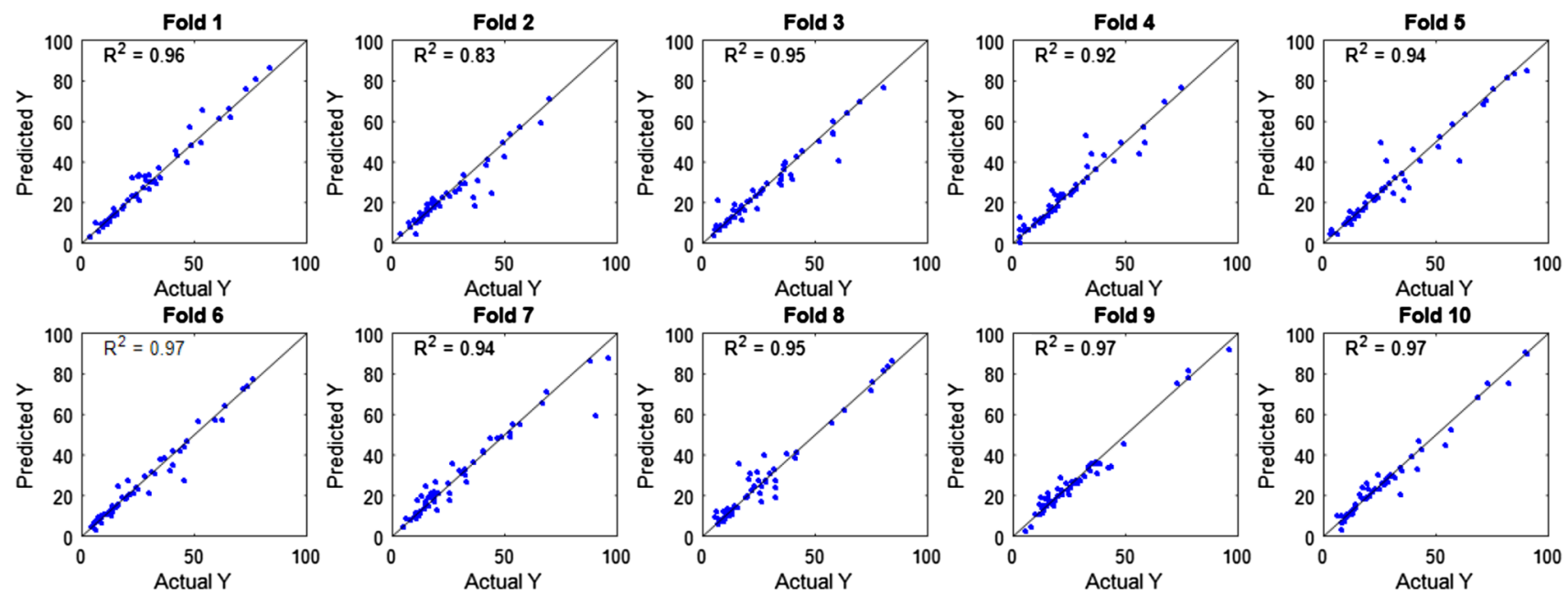

Figure 7. Testing results of the LSVR-FPA.
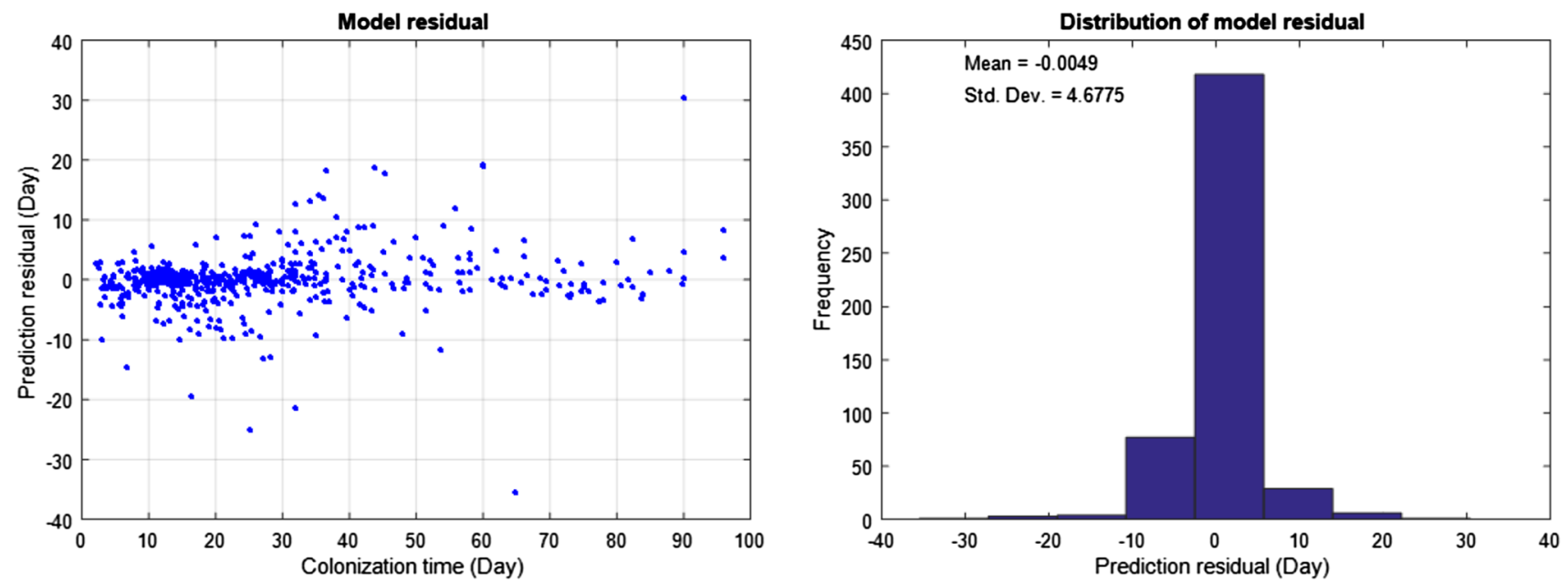

Figure 8. Residual versus actual output and the distribution of residual. 
mortar surfaces. The proposed model is based on the LSSVR as a supervised learning method for generalizing the functional mapping between the algal colonization time and a set of influencing factors (the surface roughness, porosity, surface $\mathrm{pH}$, permeability, cement type and colonization phase).

In addition, an optimization process based on the FPA is put forward to autonomously determine the hyper-parameters of the LS-SVR, namely the regularization and the RBF kernel function parameters. The hybrid approach, named as LSVR-FPA, has outperformed other two benchmark methods including the LM-ANN and the MLR. The LSVR-FPA has demonstrated desirably prediction accuracies in both training (RMSE $=2.55, R^{2}=0.98$ ) and testing phases $\left(\mathrm{RMSE}=4.55, \quad R^{2}=0.94\right)$, which are confirmed by the cross-validation process. Furthermore, the FPA deems to be very efficient for assisting the machine learning model in the task of parameter setting.

Although the prediction result obtained from the proposed model is satisfactory, the current research has a limitation in that all experiments of algal colonization are carried out in a controlled condition of temperature $\left(21 \pm 1^{\circ} \mathrm{C}\right)$ and relative humidity $(95 \pm 5 \%)$. Thus, it is of necessity to collect more data samples of algal colonization on different experimental conditions as well as on diverse building materials to enhance the generalization and the applicability of the prediction model. Therefore, the authors would like to consider these tasks to be potential extensions of the current research.

\section{References}

[1] Barberousse H, Ruot B, Yéprémian C and Boulon G 2007 An assessment of façade coatings against colonisation by aerial algae and cyanobacteria. Build. Environ. 42: 2555-2561

[2] Dalod E, Govin A, Guyonnet R, Grosseau P, Lors C and Damidot D 2014 Influence of the chemical composition of mortars on algal biofouling. In: Proceedings of the International Conference on Calcium Aluminate, May 2014, Palais des Papes, Avignon, France, pp. 523-534

[3] Gaylarde C C and Morton L H G 1999 Deteriogenic biofilms on buildings and their control: a review. Biofouling 14: 59-74

[4] Gorbushina A A 2007 Life on the rocks. Environ. Microbiol. 9: 1613-1631

[5] Scheerer S, Ortega-Morales O and Gaylarde C 2009 Microbial deterioration of stone monuments-an updated overview. Adv. Appl. Microbiol. 66: 97-139

[6] Ortega-Calvo J J, Hernandez-Marine M and Saiz-Jimenez C 1991 Biodeterioration of building-materials by cyanobacteria and algae. Int. Biodeter. 28: 165-185

[7] Shang H S, Song Y P and Qin L K 2008 Experimental study on strength and deformation of plain concrete under triaxial compression after freeze-thaw cycles. Build. Environ. 43: 1197-1204

[8] De Muynck W, Ramirez A M, De Belie N and Verstraete W 2009 Evaluation of strategies to prevent algal fouling on white architectural and cellular concrete. Int. Biodeter. Biodegr. 63: 679-689

[9] Dubosc A, Escadeillas G and Blanc P J 2001 Characterization of biological stains on external concrete walls and influence of concrete as underlying material. Cem. Concr. Res. 31: 1613-1617

[10] Rirsch E and Zhang Z 2010 Rising damp in masonry walls and the importance of mortar properties. Constr. Build. Mater. 24: 1815-1820

[11] Gaylarde C Ribas Silva M and Warscheid T 2003 Microbial impact on building materials: an overview. Mater. Struct. 36: 342-352

[12] Saiz-Jimenez C 1995 Deposition of anthropogenic compounds on monuments and their effect on airborne microorganisms. Aerobiologia 11: 161-175

[13] Cruzan R 2009 Manager's guide to preventive building maintenance. Georgia, United States: The Fairmont Press

[14] MacDonald J 1993 The effects of commercial chemical cleaning processes on sandstones in Scotland. Aberdeen: The Robert Gordon University.

[15] Burkhardt M, Kupper T, Hean S, Haag R, Schmid P, Kohler M and Boller M 2007 Biocides used in building materials and their leaching behavior to sewer systems. Water Sci. Technol. 56: 63-67

[16] Tran T H 2011 Influence des caractéristiques intrinsèques d'un mortier sur son encrassement biologique. France: Ecole Nationale Supérieure des Mines de Saint Etienne

[17] Dalod E 2015 Influence de la composition chimique de mortiers sur leur biodeterioration par les algues. France: Ecole Nationale Supérieure des Mines de Saint Etienne

[18] Tomaselli L, Lamenti G, Bosco M and Tiano P 2000 Biodiversity of photosynthetic micro-organisms dwelling on stone monuments. Int. Biodeter. Biodegr. 46: 251-258

[19] Barberousse H 2006 Etude de la diversité des algues et des cyanobactéries colonisant les revêtements de façade en France et recherche des facteurs favor isant leur implantation. Paris, France: Muséum National d'Histoire Naturelle

[20] Miller A, Dionísio A, Laiz L, Macedo M and Saiz-Jimenez C 2009 The influence of inherent properties of building limestones on their bioreceptivity to phototrophic microorganisms. Ann. Microbiol. 59: 705-713

[21] Tran T H, Govin A, Guyonnet R, Grosseau P, Lors C, Garcia-Diaz E, Damidot D, Devès O and Ruot B 2012 Influence of the intrinsic characteristics of mortars on biofouling by Klebsormidium flaccidum. Int. Biodeter. Biodegr. 70: $31-39$

[22] Tran T H, Govin A, Guyonnet R, Grosseau P, Lors C, Damidot D, Devès O and Ruot B 2013 Avrami's law based kinetic modeling of colonization of mortar surface by alga Klebsormidium flaccidum. Int. Biodeter. Biodegr. 79: $73-80$

[23] Prieto B and Silva B 2005 Estimation of the potential bioreceptivity of granitic rocks from their intrinsic properties. Int. Biodeter. Biodegr. 56: 206-215

[24] Suykens J, Gestel J V, Brabanter J D, Moor B D and Vandewalle J 2002 Least squares support vector machines. Singapore: World Scientific

[25] Yang X S 2012 Flower pollination algorithm for global optimization. In: Durand-Lose J and Jonoska N (Eds.) Unconventional computation and natural computation. BerlinHeidelberg: Springer, pp. 240-249 
[26] Xu S, Liu Z and Zhang Y 2016 Least squares support vector regression and interval type-2 fuzzy density weight for scene denoising. Soft Comput. 20(4): 1459-1470

[27] Pham A, Hoang N and Nguyen Q 2015 Predicting compressive strength of high-performance concrete using metaheuristic-optimized least squares support vector regression. J. Comput. Civil Eng. 30: 06015002

[28] Cheng M Y, Hoang N D and Wu Y W 2013 Hybrid intelligence approach based on LS-SVM and differential evolution for construction cost index estimation: a Taiwan case study. Autom. Constr. 35: 306-313

[29] Cheng M Y and Hoang N D 2013 Interval estimation of construction cost at completion using least squares support vector machine. J. Civil Eng. Manage. 20: 223-236

[30] Dos Santos G S, Luvizotto L G J, Mariani V C and Coelho L d S 2012 Least squares support vector machines with tuning based on chaotic differential evolution approach applied to the identification of a thermal process. Expert Syst. Appl. 39: 4805-4812

[31] Gadelmawla E S, Koura M M, Maksoud T M A, Elewa I M and Soliman H H 2002 Roughness parameters. J. Mater. Process. Tech. 123: 133-145

[32] De Brabanter K, Karsmakers P, Ojeda F, Alzate C, De Brabanter J, Pelckmans K, De Moor B, Vandewalle J and Suykens J A K 2010 LS-SVMlab toolbox user's guide version 1.8. Internal Report 10-146. Leuven, Belgium: ESATSISTA, K.U. Leuven

[33] Samui P and Kurup P 2012 Multivariate adaptive regression spline (MARS) and least squares support vector machine (LSSVM) for OCR prediction. Soft Comput. 16: 1347-1351

[34] Rabiei M, Arghami N, Taheri S M and Gildeh B 2014 Leastsquares approach to regression modeling in full intervalvalued fuzzy environment. Soft Comput. 18: 2043-2059

[35] Vu D T and Hoang N D 2016 Punching shear capacity estimation of frp-reinforced concrete slabs using a hybrid machine learning approach. Struct. Infrastruct. Eng. 12(9): 1153-1161

[36] Cheng M Y, Hoang N D and Wu Y W 2015 Cash flow prediction for construction project using a novel adaptive time-dependent least squares support vector machine inference model. J. Civil Eng. Manage. 21: 679-688

[37] Cheng M Y and Hoang N D 2016 A self-adaptive fuzzy inference model based on least squares SVM for estimating compressive strength of rubberized concrete. Int. J. Inf. Technol. Decis. Making 15: 603-619

[38] De Brabanter K, De Brabanter J, Suykens J A K and De Moor B 2011 Approximate confidence and prediction intervals for least squares support vector regression. IEEE Trans. Neural Netw. 22: 110-120
[39] Glover B J 2007 Understanding flowers and flowering: an integrated approach. UK: Oxford University Press

[40] Yang X S 2014 Nature-inspired optimization algorithms. Amsterdam, Netherlands: Elsevier

[41] Łukasik S and Kowalski P 2015 Study of flower pollination algorithm for continuous optimization. In: Angelov P, Atanassov K T, Doukovska L, Hadjiski M, Jotsov V, Kacprzyk J, Kasabov N, Sotirov S, Szmidt E and Zadrożny S (Eds.) Intelligent Systems'2014. Cham: Springer International Publishing, pp. 451-459

[42] Yang X S, Karamanoglu M and He X 2013 Flower pollination algorithm: a novel approach for multiobjective optimization. Eng. Optimiz. 46: 1222-1237

[43] Chakraborty D, Saha S and Dutta O 2014 DE-FPA: a hybrid differential evolution-flower pollination algorithm for function minimization. In: Proceedings of the International Conference on High Performance Computing and Applications (ICHPCA), 22-24 December 2014, Bhubaneswar, India, pp. 1-6

[44] Wang R and Zhou Y 2014 Flower pollination algorithm with dimension by dimension improvement. Math. Probl. Eng. vol. 2014, Article ID 481791

[45] Celik Y and Ulker E 2013 An improved marriage in honey bees optimization algorithm for single objective unconstrained optimization. Sci. World J., vol. 2013, Article ID 370172

[46] De B K, Suykens J and De Moor B 2013 Nonparametric regression via StatLSSVM. J. Stat. Softw. 55: 1-22

[47] Hagan M T and Menhaj M B 1994 Training feedforward networks with the Marquardt algorithm. IEEE Trans. Neural Netw. 5: 989-993

[48] Chou J S, Chiu C K, Farfoura M and Al-Taharwa I 2011 Optimizing the prediction accuracy of concrete compressive strength based on a comparison of data-mining techniques. $J$. Comput. Civil Eng. 25: 242-253

[49] Piotrowski A P and Napiorkowski J J 2011 Optimizing neural networks for river flow forecasting - Evolutionary Computation methods versus the Levenberg-Marquardt approach. J. Hydrol. 407: 12-27

[50] Tien Bui D, Pradhan B, Lofman O, Revhaug I and Dick O B 2012 Landslide susceptibility assessment in the Hoa Binh province of Vietnam: a comparison of the Levenberg-Marquardt and Bayesian regularized neural networks. Geomorphology 171-172: 12-29

[51] Farfani H A, Behnamfar F and Fathollahi A 2015 Dynamic analysis of soil-structure interaction using the neural networks and the support vector machines. Expert Syst. Appl. 42: 8971-8981

[52] Beale M H, Hagan M T and Demuth H B 2012 Neural network toolbox user's guide. United States: The MathWorks 